\title{
Reinserção da ruptura distal do bíceps braquial mediante incisão única com uso de âncoras*
}

\section{Reinsertion of Distal Brachial Biceps Tendon Rupture through Single Incision Using Anchors}

\author{
Carlos Augusto de Mattos ${ }^{1}$ Danilo Maia ${ }^{1}$ Rodolfo Castro ${ }^{1}$ Maurício Etchebehere ${ }^{2}$ () \\ ${ }^{1}$ Departamento de Ortopedia, Pontifícia Universidade Católica de \\ Campinas, Campinas, São Paulo, Brasil \\ Endereço para correspondência Carlos Augusto de Mattos, Avenida \\ Jânio da Silva Quadros 52, Bairro Jardim Chapadão, Campinas, \\ 2 Departamento de Ortopedia, Universidade Estadual de Campinas, \\ São Paulo, SP, 13070-098, Brazil (e-mail: doutormattos@yahoo.com.br).
}

\section{Resumo}

Palavras-chave

- bíceps

- cotovelo/cirurgia

- cotovelo/lesões

- ossificação heterotópica

Abstract
Objetivo Avaliar 15 pacientes com ruptura do tendão distal do bíceps submetidos a reinserção por meio de via única, anterior e transversa no antebraço com o uso de duas âncoras. Os pacientes foram submetidos a um protocolo de reabilitação e, ao término de seis meses, efetuou-se avaliação do arco de movimento do cotovelo operado e da intensidade de força durante a flexão e a supinação.

Métodos Os dados foram coletados de maneira prospectiva, e foram analisados pelo teste de Mann-Whitney e pelo teste de modelos mistos para avaliar a força entre os cotovelos operado e não operado.

Resultados Um total de $80 \%$ dos pacientes eram homens, $60 \%$ sofreram lesão do lado dominante, $46 \%$ eram trabalhadores braçais, e $73 \%$ não praticavam atividades físicas regularmente. $\mathrm{O}$ uso de anabolizante foi relatado por dois pacientes. Após o tratamento, os pacientes recuperaram $98 \%$ da força de supinação, e $94 \%$ da de flexão. De acordo com questionário de Disfunções do Braço, Ombro e Mão (Disabilities of the Arm, Shoulder and Hand, DASH), 73\% dos pacientes encontram-se dentro do esperado para uma população normal.

Conclusão A via única, anterior e transversa associada ao reparo do tendão com o uso de âncoras apresentou-se esteticamente satisfatória, com boa recuperação da força durante a flexão e a supinação, não ocorrendo casos de ossificação heterotópica ou complicações graves.

Objective To evaluate 15 patients with ruptured distal biceps tendon submitted to reinsertion via a single, anterior and transverse approach using two anchors. They were submitted to a rehabilitation protocol and, within six months, to an evaluation of the range of motion and strength intensity during flexion and supination of the operated elbow.

Trabalho realizado no Hospital Puc-Campinas, Campinas, SP, Brasil.

recebido

24 de Abril de 2018

aceito

04 de Dezembro de 2018
DOI https://doi.org/

10.1055/s-0039-3400525. ISSN 0102-3616.
Copyright $\odot 2020$ by Sociedade Brasileira License terms de Ortopedia e Traumatologia. Published by Thieme Revinter Publicações Ltda, Rio de Janeiro, Brazil 


$$
\begin{aligned}
& \text { Keywords } \\
& \text { - biceps } \\
& \text { - elbow/surgery } \\
& \text { - elbow/injuries } \\
& \text { - heterotopic } \\
& \text { ossification }
\end{aligned}
$$

\begin{abstract}
Methods The data were collected prospectively, and were analyzed through the Mann-Whitney test and the mixed-model test to evaluate the force between the operated and non-operated elbows.

Results A total of $80 \%$ of the patients were men, $60 \%$ were injured on the dominant side, $46 \%$ were manual workers, and $73 \%$ led sedentary lifestyles. The use of anabolic steroids was reported by two patients. After the treatment, the patients recovered supination strength by $98 \%$ and flexion by $94 \%$. According to the Disabilities of the Arm, Shoulder and Hand (DASH) questionnaire, $73 \%$ of the patients presented the score expected of a normal population.

Conclusion The single, anterior and transverse approach associated with tendon repair using anchors was esthetically satisfactory, with good strength recovery during flexion and supination, and no occurrence of heterotopic ossification.
\end{abstract}

\section{Introdução}

O bíceps tem uma inserção óssea distal na tuberosidade bicipital do rádio, local mais frequente para a ocorrência de rupturas. A ruptura do tendão distal bíceps braquial (TDBB) acomete principalmente o membro dominante de homens na quinta e sexta décadas de vida, usualmente no momento de contração excêntrica de flexão do cotovelo na posição de 90 graus. A degeneração tendinosa e de sua inserção podem contribuir para esse tipo de lesão, bem como o uso de anabolizantes. ${ }^{1-7}$

Após a fase aguda da lesão, há o retorno da movimentação ativa do cotovelo, mas a perda de força de flexão após a ruptura do TDBB pode chegar a 30\%, enquanto na supinação pode ocorrer perda mais acentuada (cerca de $40 \%$ ), pois o bíceps é considerado biomecanicamente como supinador primário do antebraço e flexor secundário do cotovelo. ${ }^{7-11}$

Por esse motivo, a reparação cirúrgica com reinserção anatômica do TDBB na tuberosidade do radial é indicada para atletas, pacientes ativos com alta demanda, e também para aqueles que não se conformam com déficits funcionais permanentes ou com a deformidade estética. ${ }^{11-14}$

Existem várias técnicas cirúrgicas descritas para o reparo da ruptura do TDBB, sendo a primeira delas por meio de uma incisão anterior única atravessando o cotovelo. Outras técnicas já foram descritas posteriormente, como dupla incisão anterior e dupla mini-incisão, sendo uma anterior e outra posterolateral. A literatura tem demonstrado nessas técnicas complicações como lesão da artéria radial, lesão do nervo mediano, lesão do nervo interósseo posterior, ou sinostose radioulnal proximal. ${ }^{2,4,6,8,12-17} \mathrm{O}$ profundo conhecimento da anatomia local minimiza as complicações pós-operatórias. Em razão disso, a via de acesso anterior transversa na região da tuberosidade do rádio é mais anatômica e respeita as linhas de forças regionais, além de não atravessar a prega anterior do cotovelo, evitando retrações cicatriciais. Outra vantagem é não atravessar a membrana interóssea, diminuindo chances de sinostoses ou perda de pronossupinação do antebraço.

O objetivo deste trabalho é realizar avaliação pós-operatória funcional da reinserção cirúrgica do TDBB em lesões agudas por meio de uma via única transversa anterior na região da tuberosidade bicipital do rádio, comparando-a com outras técnicas já descritas.

\section{Materiais e Métodos}

Foi realizado estudo prospectivo, aprovado pelo comitê de ética (CAAE 23700313.4.0000.5481), com autorização e assinatura do termo de consentimento livre e esclarecido por todos os pacientes. No período de 2014 a 2017, foram operados consecutivamente 15 pacientes, 12 homens e 3 mulheres, com média de idade de 44 anos (entre 26 e 65 anos), diagnosticados com ruptura total do TDBB, confirmada por meio da anamnese, do exame físico, e de dois exames de imagens (radiografias do cotovelo acometido em incidência anteroposterior e de perfil, e ressonância magnética) realizados no mesmo serviço de imagem e no mesmo aparelho de ressonância magnética. Os pacientes foram atendidos no ambulatório do pesquisador principal. Os sujeitos foram incluídos no protocolo e operados no mesmo hospital pelo mesmo pesquisador. Todos os pacientes tinham o braço direito como membro dominante, e em nove pacientes o lado dominante estava acometido. Dois pacientes declararam uso regular de anabolizante.

Foram definidos os seguintes critérios de inclusão: pacientes maiores de 18 anos, com lesão completa do TDBB por esforço não traumático, lesão aguda (menos de quatro semanas), e unilateral. Foram excluídos pacientes com lesões com mais de quatro semanas de duração, lesões parciais do bíceps, lesões ou fraturas prévias nos membros superiores (ipsilaterais ou contralaterais), e aqueles que não concordaram com o tratamento cirúrgico ou com o termo de consentimento.

\section{Técnica Cirúrgica}

Os pacientes são submetidos a anestesia geral associada a bloqueio de plexo braquial, com membro superior posicionado a cerca de 90 graus de abdução. É instalado garrote pneumático (pressão média de $250 \mathrm{mmHg}$ ) na porção superior do braço, com o antebraço e o cotovelo em extensão e supinação completa. 
A incisão de pele é realizada a $3 \mathrm{~cm}$ distal e paralelamente à prega do cotovelo, e transversalmente ao eixo do antebraço, com cerca de $4 \mathrm{~cm}$ de extensão. Encontra-se o plano intermuscular entre a porção medial do músculo braquiorradial e lateralmente ao pronador redondo. Afastam-se esses músculos com afastadores tipo Langenbeck para evitar compressões de nervos contra o osso do rádio, principalmente o nervo interósseo posterior. A artéria radial passa dorsalmente ao músculo braquiorradial. 0 nervo cutâneo lateral do antebraço localiza-se lateralmente à veia cefálica, que é facilmente identificada.

Com o antebraço em supinação completa, visualizamos a tuberosidade bicipital do rádio, sendo que em alguns casos é possível observar o coto distal do bíceps. Realizamos cruentização e decorticação na região da inserção anatômica do bíceps ulnalmente à tuberosidade bicipital e preservando a sua anatomia.

Pela mesma incisão, proximalmente identifica-se o coto proximal da lesão, sendo feito o reparo distal com fio multifilamentar sintético absorvível número 2 .

Com o tendão e o leito ósseo preparados, introduzimos 2 âncoras de titânio de $4 \mathrm{~mm}$ de diâmetro, carregadas com fios duplos de alta resistência na região cruentizada do osso, com cerca de $0,5 \mathrm{~cm}$ de distância entre elas para evitar fraturas adicionais.

Iniciamos a sutura pela âncora mais distal, transfixando a região mais distal do coto do tendão, aproximando-o da parte mais distal do leito cruento, utilizando um ponto no qual o fio não desliza no tendão (ponto de autotravamento). No momento da tenodese, flexionamos o antebraço a cerca de 30 graus para diminuir a tensão da sutura. Após esse ponto, realizamos pontos simples, promovendo o maior contato possível do tendão com o osso cruento.

Da primeira à quarta semanas, os pacientes foram orientados a manterem o braço na posição anatômica, com o cotovelo fletido a 90 graus em tipóia. Na quarta semana do pós-operatório, foram encaminhados à reabilitação.

Foram realizadas radiografias nas incidências anteroposterior e de perfil do cotovelo no pós-operatório imediato para controle da colocação das âncoras, com um mês e seis meses após a cirurgia, para avaliação também da presença de ossificação heterotópica (- Figura 1A e 1B).
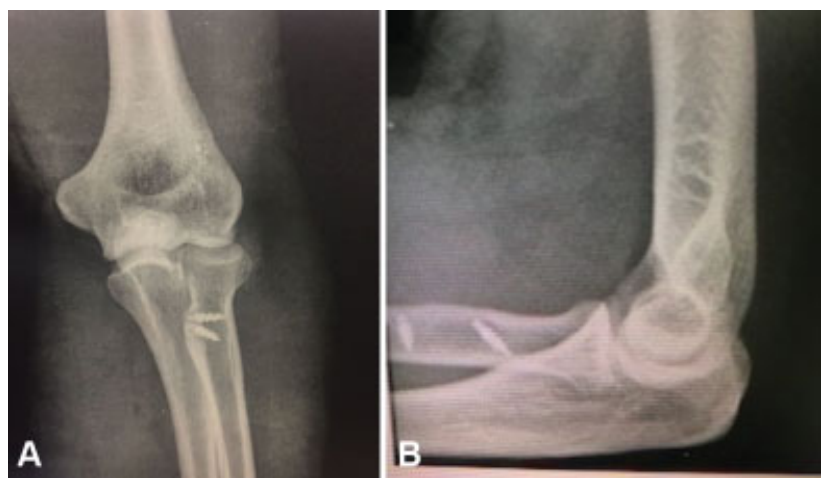

Fig. 1 Caso 7, sexo masculino, 40 anos, radiografia pós-operatória. (A) Anteroposterior; (B) perfil.
A avaliação funcional pós-operatória foi realizada aos seis meses, por dois médicos avaliadores diferentes do cirurgião, por meio de testes com mensuração de forças musculares com dinamômetro isométrico digital (Lafayette Manual Muscle Testing System 01165, Lafayette Instrument, Lafayette, IN, EUA) ${ }^{18-20}$ e escala específica (questionário de Disfunções do Braço, Ombro e Mão [Disabilities of the Arm, Shoulder and Hand, DASH]), ${ }^{21}$ além da avaliação do arco de movimento (ADM) do cotovelo lesionado e do não lesionado utilizando-se de um goniômetro, com a medida da flexão e extensão, e da supinação e pronação.

Todos os pacientes foram submetidos a avaliação da força muscular do bíceps braquial por meio de dinamômetro isométrico digital ${ }^{18-20}$ (Lafayette Instrument), sendo aferida a força máxima de flexão com o cotovelo em flexão de 90 graus, e a força máxima de supinação com o antebraço na posição neutra. Para evitar viés, usou-se uma barra com marcação como referência, utilizada pelo mesmo examinador em todos os casos (-Figuras 2 e 3). Foram feitas quatro medidas em flexão e em supinação do cotovelo lesionado e do não lesionado. $O$ intervalo entre as aferições foi de um minuto (-Figuras 4 e $\mathbf{5}$ ). A primeira medida de cada teste foi

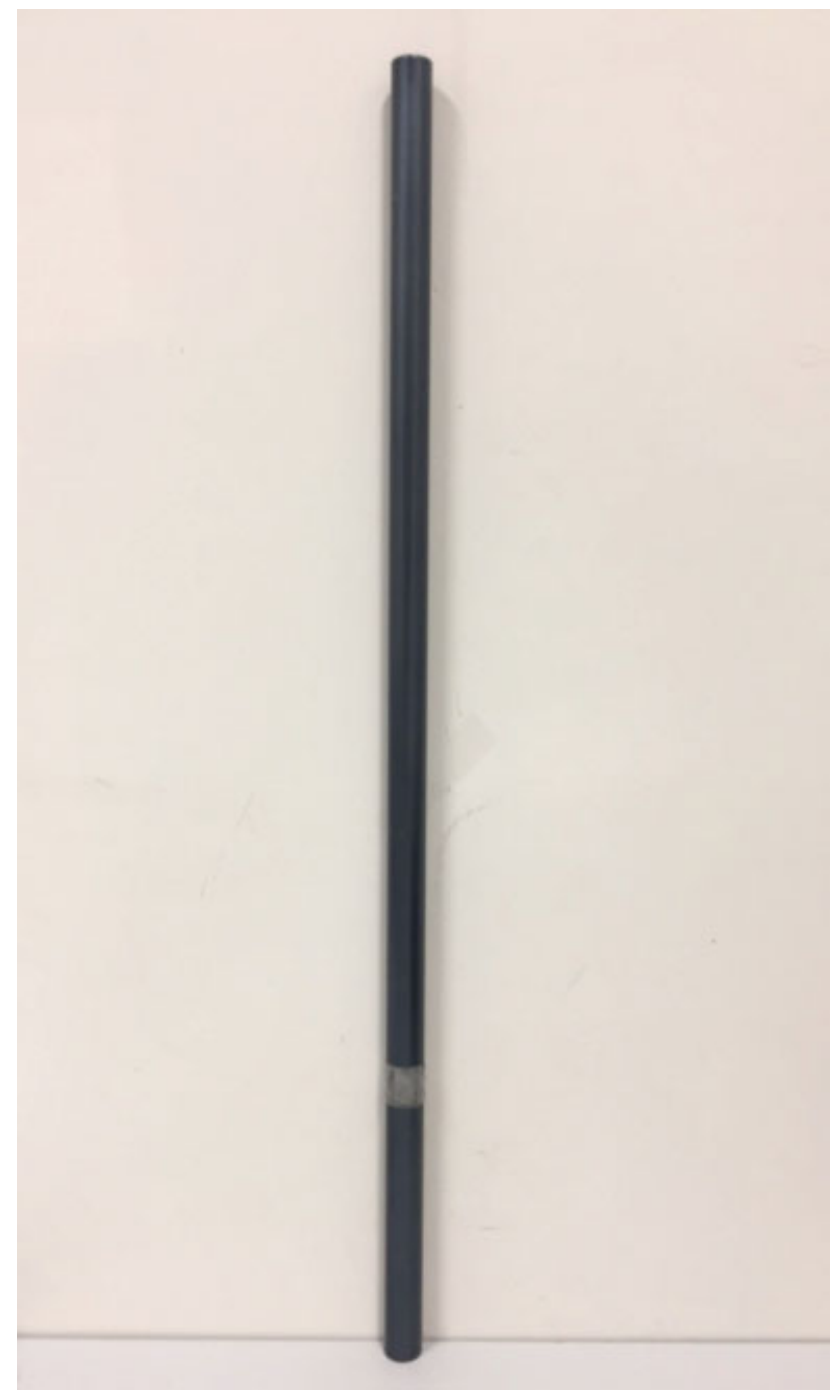

Fig. 2 Barra acessória para dinamômetro. 


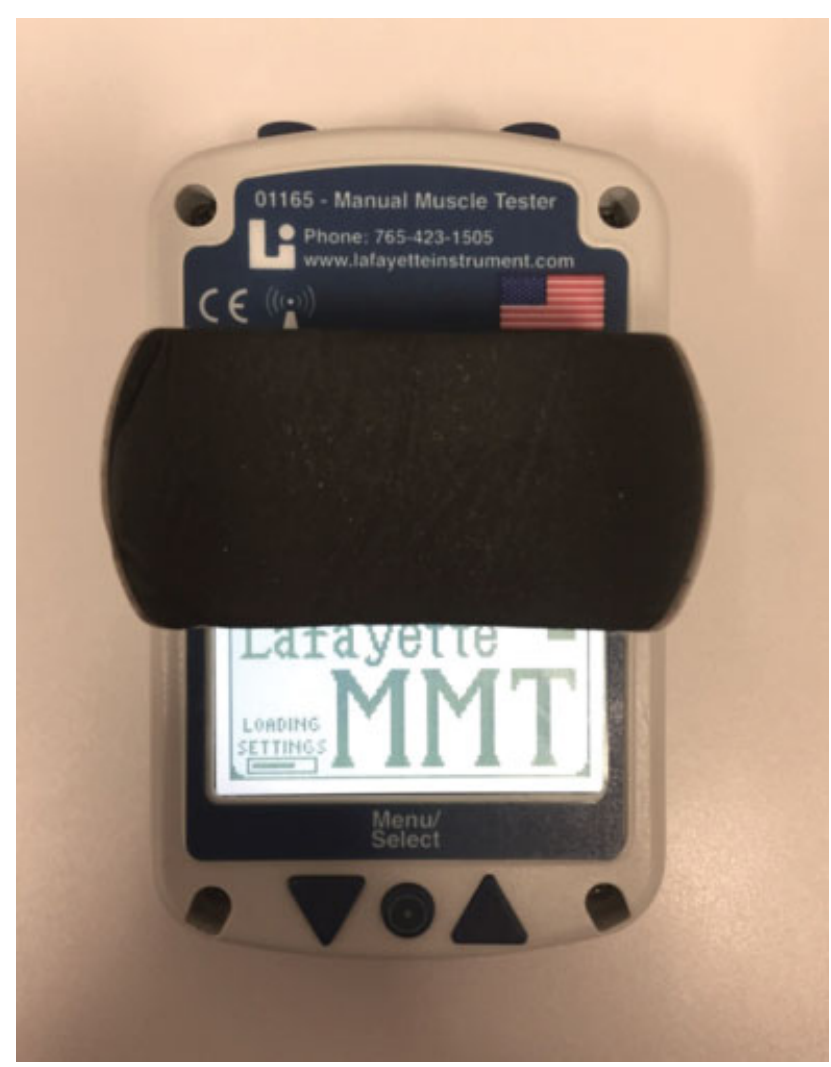

Fig. 3 Dinamômetro digital.

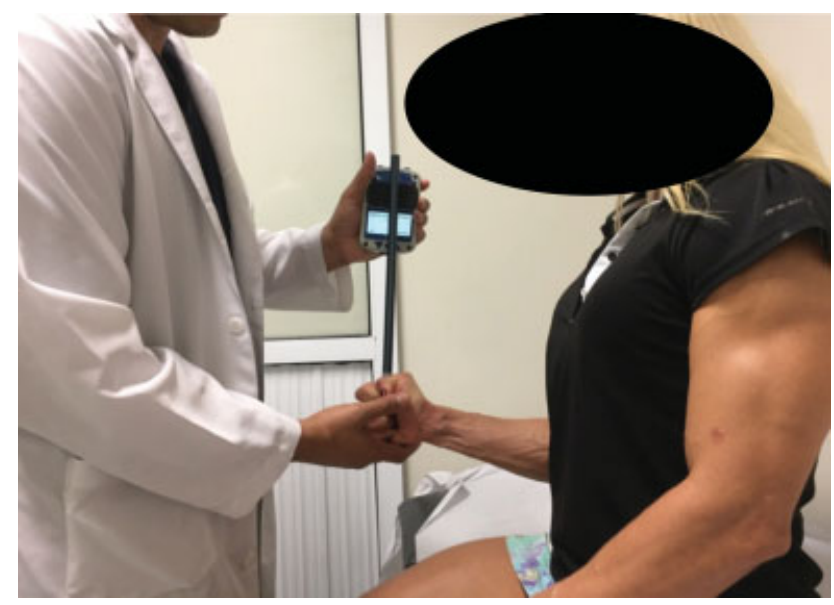

Fig. 4 Caso 13, sexo feminino, 35 anos. Teste de força em supinação.

descartada, pois serviu como treinamento para o paciente. As demais foram utilizadas para os cálculos estatísticos, em que se utilizou a média dos valores.

Para pontuação do resultado funcional pós-operatório, utilizou-se o questionário $\mathrm{DASH}^{21}{ }^{21}$ no qual pontuações próximas a " 0 " indicam menores limitações e, portanto, melhor resultado técnico-cirúrgico, enquanto a pontuação "100" indica maior limitação e, consequentemente, pior resultado.

As complicações pós-operatórias foram investigadas e registradas.

As variáveis idade, pontuação no DASH, complicações, ADM passivo, força de flexão e supinação foram analisadas de maneira descritiva usando o programa Numbers (Apple Inc.,

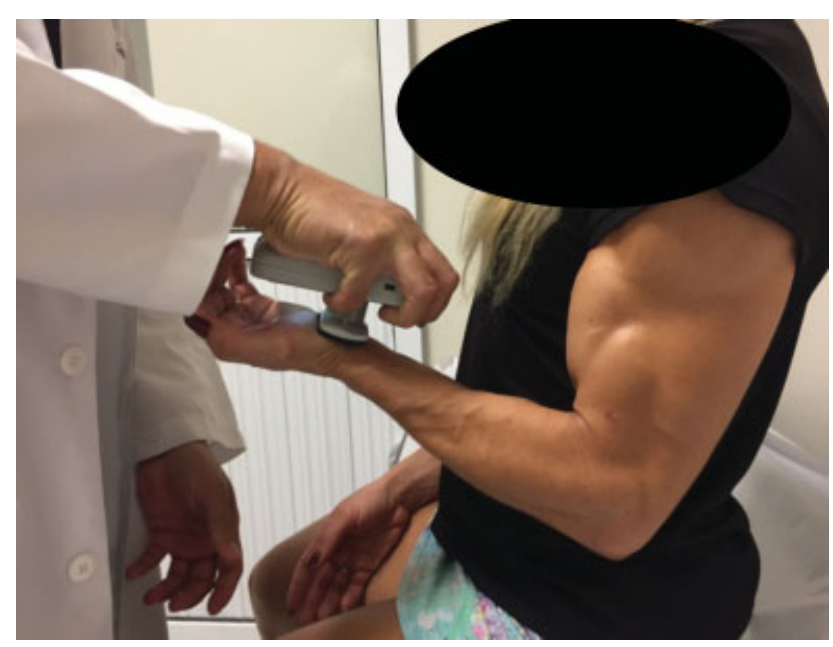

Fig. 5 Caso 13, sexo feminino,35 anos. Teste de força em flexão.

Cupertino, CA, EUA). Utilizou-se o teste de Mann-Whitney para comparar o ADM entre o cotovelo operado e o não operado, e o teste de modelos mistos para avaliar a força entre o cotovelo operado e o não operado, considerando-se estatisticamente significativos valores de $p<0,005$.

\section{Resultados}

A média da força aferida na flexão do cotovelo operado foi de $20,65 \mathrm{kgf}$, e de 22,02 kgf no cotovelo não operado ( $p=0,904)$, enquanto, na supinação, a média foi de $2,59 \mathrm{kgf}$ no cotovelo operado, e de 2,64 kgf no não operado ( $p=0,668$ ), e observamos que não houve diferença significativa entre as forças dos braços operados e não operados.

A média do ADM ativo de flexão do cotovelo operado foi de 134,67 graus, e de 136 graus no cotovelo não operado, enquanto a média da supinação foi de 69,47 graus no cotovelo operado, e de 79,33 no não operado. Para comparar os valores desses ângulos, utilizamos o teste de MannWhitney, e, neste caso, observamos que não houve diferença significativa $(p>0,05)$ nos ângulos dos braços operados e não operados em nenhum dos dois movimentos realizados, sendo $p=0,345$ para flexão, e $p=0,114$ para supinação (-Tabela 1).

Em relação à dominância do braço acometido, também não foram encontradas diferenças significativas entre as médias de força e angulação dos cotovelos operados e não operados nos exercícios realizados, independente de se o braço acometido era dominante ou não dominante (-Tabela $\mathbf{2}$ ).

Com relação às complicações observadas, ocorreu um caso de soltura de uma âncora após o período de cicatrização

Tabela 1 Comparação da média do arco de movimento dos cotovelos operados e não operados

\begin{tabular}{|l|l|l|l|}
\hline $\begin{array}{l}\text { Arco de } \\
\text { movimento }\end{array}$ & Operado & $\begin{array}{l}\text { Não } \\
\text { operado }\end{array}$ & Valor de $\boldsymbol{p}$ \\
\hline Flexão & 134,67 & 136 & 0,345 \\
\hline Supinação & 69,47 & 79,33 & 0,114 \\
\hline
\end{tabular}


Tabela 2 Comparação de força (média) e arco de movimento (ADM) entre os braços dominante e não dominante

\begin{tabular}{|c|c|c|c|c|}
\hline & & $\begin{array}{l}\text { Membro } \\
\text { dominante }\end{array}$ & $\begin{array}{l}\text { Membro } \\
\text { não dominante }\end{array}$ & Valor de $p$ \\
\hline \multirow{5}{*}{$\begin{array}{l}\text { Braço dominante } \\
\text { com lesão } \\
n=9\end{array}$} & Supinação & 2,51 & 2,41 & 0,86 \\
\hline & Flexão & 20,28 & 20,67 & 0,93 \\
\hline & ADM - flexão & 134,44 & 136,67 & 0,37 \\
\hline & ADM - supinação & 63,33 & 78,89 & 0,20 \\
\hline & ADM - pronação & 72,22 & 75,00 & 1,00 \\
\hline \multirow{5}{*}{$\begin{array}{l}\text { Braço não dominante com lesão } \\
n=6\end{array}$} & Supinação & 2,7 & 2,99 & 0,62 \\
\hline & Flexão & 21,21 & 24,06 & 0,57 \\
\hline & ADM - flexão & 135 & 135,00 & 1,00 \\
\hline & ADM - supinação & 78,67 & 80,00 & 0,37 \\
\hline & ADM - pronação & 75,00 & 73,67 & 1,00 \\
\hline
\end{tabular}

do tendão, detectado na radiografia com seis meses de pósoperatório, que não interferiu no resultado cirúrgico.

Outro caso apresentou uma neuroapraxia do radial do tipo “alta”, ou seja, com paralisia total do nervo radial, temporária, com resolução espontânea após três meses.

\section{Discussão}

A casuística de nosso trabalho assemelha-se à casuística mundial, na qual a ruptura do TDBB é mais comum em homens entre a quarta e quinta décadas de vida e no membro dominante. .,6 $^{3}$

Na literatura, várias técnicas cirúrgicas foram descritas para o tratamento da lesão do TDBB, sendo a primeira delas por meio de uma incisão anterior única atravessando o cotovelo. Outras técnicas mais recentes foram descritas e amplamente utilizadas, como dupla incisão anterior e dupla mini-incisão, não havendo consenso na literatura sobre a superioridade de uma delas em estudos clínicos publicados até o momento. Todas as técnicas têm apresentado complicações: lesão da artéria radial, lesão do nervo interósseo posterior, ossificação heterotópica, ou sinostose radioulnal proximal. ${ }^{2,4,6,8,12-17}$

Nós escolhemos utilizar a via de acesso única anterior do antebraço de cerca de $4 \mathrm{~cm}$ sobre a tuberosidade anterior do rádio porque ela possibilita minimizar a dissecção dos tecidos moles durante o reparo, pois não atravessa a sindesmose, e apresenta acesso direto ao local de inserção do TDBB, permitindo a reinserção ulnal mais anatômica, preservando a tuberosidade. ${ }^{8,15,22,23}$ Além disso, apresenta bom resultado estético cicatricial (- Figura 6).

Schmidt et al, ${ }^{9}$ em seu trabalho anatômico com estudo biomecânico, demonstram que a preservação da tuberosidade bicipital radial tem um efeito "cam" no momento de força de supinação, e o local anatômico de reinserção distal do bíceps encontra-se ulnalmente à essa tuberosidade. Em estudo biomecânico em cadáveres, a reinserção anatômica apresenta os mesmos resultados biomecânicos do tendão íntegro em relação à supinação, sendo os piores resultados quando a reinserção é feita radialmente à tuberosidade, sendo esta última considerada não anatômica. Nessa situação, quando compa-

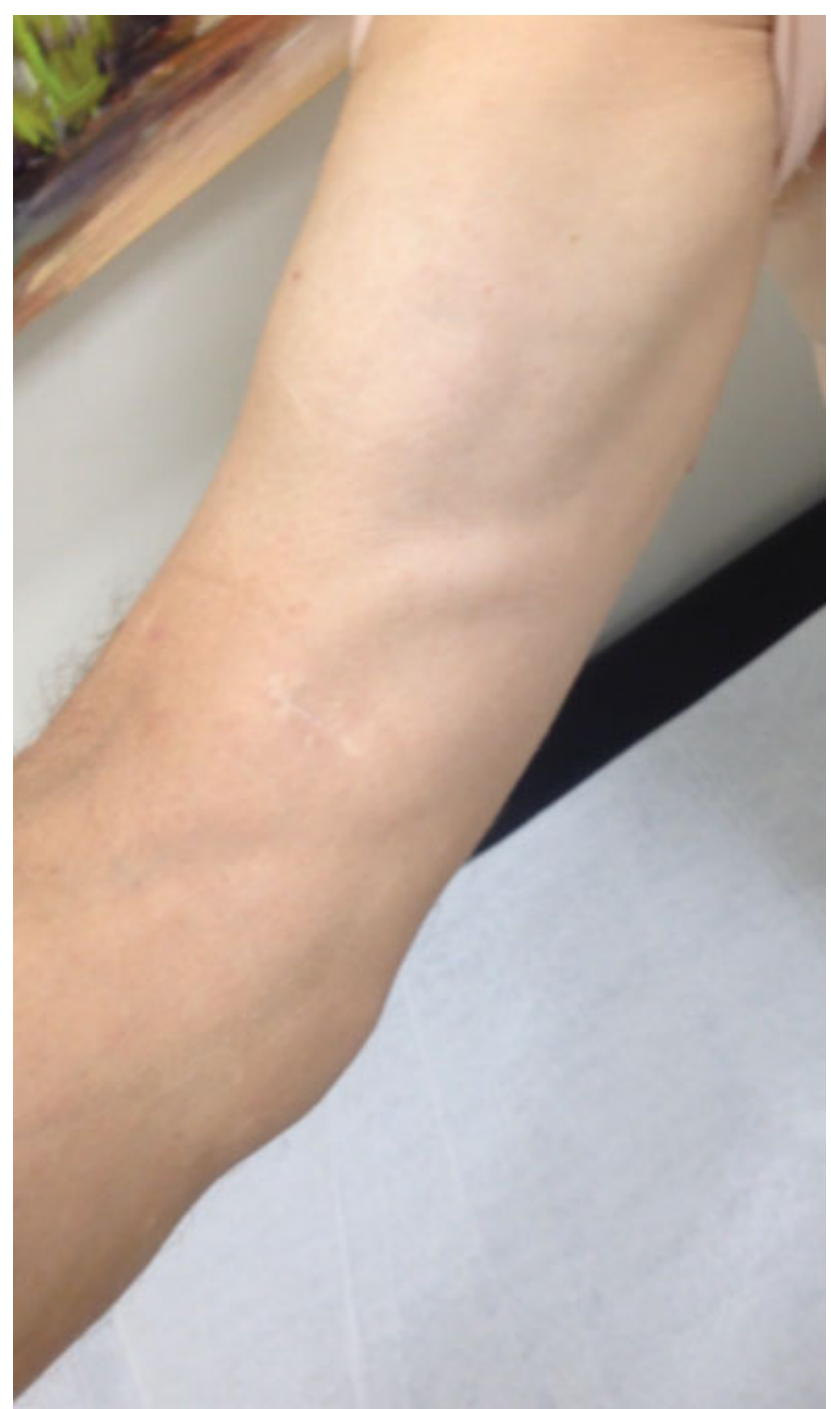

Fig. 6 Caso 8, sexo masculino, 59 anos. Aspecto pós-operatório tardio.

rada com a reinserção anatômica, há um déficit biomecânico de força de $15 \%$ da supinação com o antebraço em posição neutra, com aumento para $40 \%$ de déficit quando o antebraço é testado em 45 graus de supinação. ${ }^{10}$ 
Em nossa casuística, os pacientes foram avaliados aos seis meses de pós-operatório, e todos os participantes responderam ao questionário DASH. ${ }^{21}$ Apesar de a média de 18,66 $(3,3-76,6)$ pontos no DASH ter sido maior do que a encontrada em outros estudos ${ }^{6,11,14,22,23}(2,88-7,9)$, a maioria dos pacientes (73\%) encontrava-se dentro do esperado para uma população normal de acordo com o questionário DASH.

A média do ADM dos cotovelos operados em comparação aos não operados não apresentou diferença significativa, tanto na flexo-extensão quanto na pronossupinação. A média para flexão do cotovelo foi de 134 graus, semelhante à de outros trabalhos, assim como observamos média de 73 graus de pronação e 69 graus de supinação, que foram valores próximos àqueles de outros artigos, nos quais a pronação variou de 75 a 82 graus, e a supinação, de 62 a 88 graus. ${ }^{6,11,14,22-24}$ Um déficit angular de até 10 graus para supinação, em relação ao membro contralateral, não compromete as atividades de vida diária da maioria dos pacientes, sendo que outros estudos com técnicas semelhantes encontraram déficit de 3 a 6,4 graus em média. $2,4,6,8-14,22-24$

Segundo a literatura, a reinserção cirúrgica pode recuperar a função e forças musculares de flexão e supinação semelhantes as do membro não operado. 0 tratamento conservador, segundo Stoll e Huang, ${ }^{2}$ apresenta perdas de pico de força de $21 \%$ a $55 \%$ de supinação, $79 \%$ de resistência na supinação, $10 \%$ a $40 \%$ de pico de força da flexão, e $30 \%$ de resistência na flexão.

Inúmeros implantes e técnicas têm sido utilizados para a tenodese do bíceps braquial, em diferentes ensaios biomecânicos, sendo botão metálico, parafuso de interferência, âncora e suturas transósseas os mais utilizados, todos superiores à força necessária para a ruptura do TDBB, que é de 204 N. ${ }^{1}$ Segundo estudo biomecânico de Mazzocca et al, ${ }^{1}$ em ordem decrescente de força de resistência contra arrancamento (medida em N), tem-se o botão metálico (440 N), a âncora $(381 \mathrm{~N})$, o túnel transósseo $(310 \mathrm{~N})$, e o parafuso de interferência $(232 \mathrm{~N})$. Porém, implantes que atravessam o rádio, como o botão metálico, fios guias de parafusos de interferências, ou mesmo as suturas transósseas, podem causar lesões de estruturas neurovasculares ao atravessar o osso. Além disso, estudos clínicos que comparam âncoras com os demais implantes têm demonstrado bons resultados com as âncoras. ${ }^{3,8,13,15,22,24}$ Em nosso meio, as âncoras têm sido amplamente utilizadas em outros tipos de procedimentos, como no ombro, pois são fornecidas pelo Sistema Único de Saúde (SUS), assim como o parafuso de interferência, ao contrário do botão metálico, que não está na lista oficial de implantes oferecidos pelo SUS, denominado Sistema de Gerenciamento da Tabela de Procedimentos, Medicamentos e Órteses/Proteses e Materiais Especiais (OPM) do SUS (Sigtap). ${ }^{25}$ Em relação aos custos, constam na lista do Sigtap da competência de outubro/18, o valor de $\mathrm{R} \$ 486,29$ para o parafuso de interferêrencia, e de $\mathrm{R} \$ 197,66$ para a âncora, sendo, portanto, este último o implante com o menor custo disponível no sistema público de saúde em nosso país. Os pontos transósseos, apesar do menores custos, necessitam de uma maior exposição e apresentam o inconveniente já relatado da necessidade de atravessar o osso, com risco aumentado de lesões neurológicas. Por todos esses fatores, optamos pelo uso de âncoras com tenodese na posição anatômica após escarificação de um leito ulnal na tuberosidade do rádio, semelhante às técnicas de tenodese do manguito rotador ou do bíceps proximal com âncoras.

Utilizamos o dinamômetro digital isométrico devido à sua praticidade e viabilidade de uso como instrumento de aferição ambulatorial de força. ${ }^{18-20} \mathrm{Na}$ nossa amostra, os pacientes não apresentaram diferença significativa entre os membros em relação à força. Em média, as forças de supinação e de flexão foram restauradas em $98 \%$ e 94\%, respectivamente, o que se considera satisfatório, pois, segundo revisão sistemática publicada por Chavan et al, ${ }^{23}$ funcionalmente, um bom resultado deve restaurar no mínimo $80 \%$ da força de flexão e de supinação e reduzir no máximo 30 graus em qualquer ADM do cotovelo (flexão, extensão, supinação ou pronação). Quanto à recuperação da força do membro operado, resultados semelhantes foram encontrados em outros estudos com diferentes técnicas, variando de $80 \%$ até $95 \%$ para supinação, e de $90 \%$ até $95 \%$ para flexão do cotovelo. $2,6,8,12-14,23$

O desenvolvimento de ossificação heterotópica não foi constatado no pós-operatório dos 15 pacientes avaliados, mesmo não sendo adotado pelo grupo o uso rotineiro de anti-inflamatórios não hormonais, pois essa profilaxia pode aumentar o risco de sangramento gastrointestinal. Uma soltura de âncora ocorreu durante o processo de recuperação, mas isso não afetou negativamente o resultado objetivo dos testes, representando um índice de 6,6\% de complicações, semelhante aos estudos publicados na literatura. 4,8,12-15,23 Apesar do manuseio cuidadoso dos afastadores e a frequente liberação da tensão de partes moles do lado radial durante o procedimento cirúrgico, um paciente apresentou neuropraxia do radial, com características de déficit motor do tipo alto nervo radial, ou seja, proximal ao local da cirurgia, e foi associado ao uso de garrote, com resolução total espontânea em três meses, não sendo considerada uma complicação direta da técnica cirúrgica.

Em suma, ao utilizar a via única anterior com reparo anatômico do TDBB com âncoras, um procedimento minimamente invasivo e de baixo custo, constatou-se que os pacientes evoluíram bem, com alto índice de satisfação e mínima perda do ADM em alguns casos. Dessa forma, demonstramos tratar-se de uma técnica segura e eficaz para esse tipo de lesão.

\section{Conclusões}

A via única anterior transversa do antebraço com reparo anatômico do TDBB com duas âncoras demonstrou bons resultados clínicos e baixo índice de complicações. É uma técnica com resultado funcional semelhante ao de outras técnicas descritas, e com mínima alteração estética.

Conflito de Interesses

Os autores declaram não haver conflito interesses. 


\section{Referências}

1 Mazzocca AD, Cohen M, Berkson E, et al. The anatomy of the bicipital tuberosity and distal biceps tendon. J Shoulder Elbow Surg 2007;16(01):122-127

2 Stoll LE, Huang JI. Surgical Treatment of Distal Biceps Ruptures. Orthop Clin North Am 2016;47(01):189-205

3 Schmidt CC, Savoie FH 3rd, Steinmann SP, et al. Distal biceps tendon history, updates, and controversies: from the closed American Shoulder and Elbow Surgeons meeting-2015. J Shoulder Elbow Surg 2016;25(10):1717-1730

4 Guglielmino C, Massimino P, Ioppolo F, Castorina S, Musumeci G, Di Giunta A. Single and dual incision technique for acute distal biceps rupture: clinical and functional outcomes. Muscles Ligaments Tendons J 2016;6(04):453-460

5 Haverstock J, Athwal GS, Grewal R. Distal biceps injuries. Hand Clin 2015;31(04):631-640

6 Giacalone F, Dutto E, Ferrero M, Bertolini M, Sard A, Pontini I. Treatment of distal biceps tendon rupture: why, when, how? Analysis of literature and our experience. Musculoskelet Surg 2015;99(01, Suppl 1):S67-S73

7 An KN, Hui FC, Morrey BF, Linscheid RL, Chao EY. Muscles across the elbow joint: a biomechanical analysis. J Biomech 1981;14 (10):659-669

8 Dunphy TR, Hudson J, Batech M, Acevedo DC, Mirzayan R. Surgical Treatment of Distal Biceps Tendon Ruptures: An Analysis of Complications in 784 Surgical Repairs. Am J Sports Med 2017; 45(13):3020-3029

9 Schmidt CC, Brown BT, Williams BG, et al. The importance of preserving the radial tuberosity during distal biceps repair. J Bone Joint Surg Am 2015;97(24):2014-2023

10 Prud'homme-Foster M, Louati H, Pollock JW, Papp S. Proper placement of the distal biceps tendon during repair improves supination strength-a biomechanical analysis. J Shoulder Elbow Surg 2015;24(04):527-532

11 Schmidt CC, Brown BT, Sawardeker PJ, DeGravelle M Jr, Miller MC. Factors affecting supination strength after a distal biceps rupture. J Shoulder Elbow Surg 2014;23(01):68-75

12 Witkowski J, Królikowska A, Czamara A, Reichert P. Retrospective evaluation of surgical anatomical repair of distal biceps brachii tendon rupture using suture anchor fixation. Med Sci Monit 2017; 23:4961-4972

13 Waterman BR, Navarro-Figueroa L, Owens BD. Primary repair of traumatic distal biceps ruptures in a military population: clinical outcomes of single- versus 2-incision technique. Arthroscopy 2017;33(09):1672-1678

14 Suda AJ, Prajitno J, Grützner PA, Tinelli M. Good isometric and isokinetic power restoration after distal biceps tendon repair with anchors. Arch Orthop Trauma Surg 2017;137(07):939-944

15 Maciel RA, Costa PS, Figueiredo EA, Belangero PS, Pochini AC, Ejnisman B. Acute distal biceps ruptures: single incision repair by use of suture anchors. Rev Bras Ortop 2017;52(02):148-153

16 Boyd HB, Anderson LD. A method for reinsertion of the distal biceps brachii tendon. J Bone Joint Surg Am 1961;43:1041-1043

17 Hartman MW, Merten SM, Steinmann SP. Mini-open 2-incision technique for repair of distal biceps tendon ruptures. J Shoulder Elbow Surg 2007;16(05):616-620

18 Karnofel H, Wilkinson K, Lentell G. Reliability of isokinetic muscle testing at the ankle. J Orthop Sports Phys Ther 1989;11(04): $150-154$

19 Schwartz S, Cohen ME, Herbison GJ, Shah A. Relationship between two measures of upper extremity strength: manual muscle test compared to hand-held myometry. Arch Phys Med Rehabil 1992; 73(11):1063-1068

20 Reed RL, Den Hartog R, Yochum K, Pearlmutter L, Ruttinger AC, Mooradian AD. A comparison of hand-held isometric strength measurement with isokinetic muscle strength measurement in the elderly. J Am Geriatr Soc 1993;41(01):53-56

21 Orfale AG, Araújo PM, Ferraz MB, Natour J. Translation into Brazilian Portuguese, cultural adaptation and evaluation of the reliability of the Disabilities of the Arm, Shoulder and Hand Questionnaire. Braz J Med Biol Res 2005;38(02):293-302

22 Shields E, Olsen JR, Williams RB, Rouse L, Maloney M, Voloshin I. Distal biceps brachii tendon repairs: a single-incision technique using a cortical button with interference screw versus a doubleincision technique using suture fixation through bone tunnels. Am J Sports Med 2015;43(05):1072-1076

23 Chavan PR, Duquin TR, Bisson LJ. Repair of the ruptured distal biceps tendon: a systematic review. Am J Sports Med 2008;36 (08):1618-1624

24 Sutton KM, Dodds SD, Ahmad CS, Sethi PM. Surgical treatment of distal biceps rupture. J Am Acad Orthop Surg 2010;18(03): 139-148

25 Brasil. Ministério da Saúde;2013SIGTAP-Sistema de Gerenciamento da Tabela de Procedimentos, Medicamentos e OPM do SUS [acesso em 2018 out 14]. Disponível em: http://sigtap. datasus.gov.br/tabela-unificada/app/sec/inicio.jsp 